\title{
The ultrasonic harmonic scalpel for circumcision: experimental evaluation using dogs
}

\begin{abstract}
Mou Peng*, Zhe Meng*, Zhong-Hua Yang and Xing-Huan Wang
Male circumcision is one of the most commonly performed operations worldwide, and many novel techniques have been developed for better postoperative outcomes. The purpose of this study was to explore the feasibility of applying the ultracision harmonic scalpel (UHS) for circumcision by using dogs. Sixteen adult male dogs were divided into two groups: the UHS group and the control group. The dogs were circumcised with either the UHS or a conventional scalpel. The UHS circumcision procedure and the effects were imaged 1 week after surgery. The two groups were compared with respect to the operative time and volume of blood loss. Postoperative complications, including oedema, infection, bleeding of the incision and wound dehiscence, were recorded for both groups. The mean operative time for the UHS group was only $5.1 \mathrm{~min}$ compared with the $35.5 \mathrm{~min}$ of the conventional group. The mean blood loss was less than $2 \mathrm{ml}$ for the UHS group and $15 \mathrm{ml}$ for the conventional group. There was only one case of mild oedema in the UHS group, but the postoperative complications in the conventional group included two cases of mild oedema, one infection of the incision and one case of bleeding of the incision. In conclusion, circumcision using UHS is a novel technique to treat patients with phimosis and excessive foreskin, and this method has a short operative time, less blood loss and fewer complications than the conventional scalpel method. This small animal study provides a basis for embarking on a larger-scale clinical trial of the UHS.
\end{abstract}

Asian Journal of Andrology (2013) 15, 93-96; doi:10.1038/aja.2012.67; published online 8 October 2012

Keywords: conventional circumcision; dog; electrocautery technique; Plastibell device; ultracision harmonic scalpel

\section{INTRODUCTION}

Male circumcision is one of the most commonly performed operations worldwide. ${ }^{1,2}$ Approximately $30 \%$ of men globally and $35 \%$ of men in developing countries are circumcised for religious, cultural, medical and other reasons, such as hygiene, aesthetics and peer pressure. ${ }^{3,4}$ Circumcision prevents not only urinary tract infections in infants, but also sexually transmitted diseases and cervical and penile cancer in adults. ${ }^{5,6}$ Furthermore, the World Health Organization, the Joint United Nations Programme on HIV/AIDS and the US President's Emergency Plan for AIDS Relief have identified male circumcision as an effective means of HIV prevention in regions with high rates of heterosexual transmission. ${ }^{7-10}$

Nowadays, the main techniques used in circumcision are conventional circumcision and the Plastibell device. However, the current techniques in circumcision have some disadvantages. Furthermore, patients want to choose the operation that has the least pain and shortest operative time. Therefore, novel techniques of male circumcision are required.

The ultracision harmonic scalpel (UHS) has been widely used in laparoscopic surgery and is reported to be safe and quick for the cutting and coagulating of tissue. To determine whether the use of the UHS could have some advantages in male circumcision, we used the UHS to remove excessive foreskin in dogs, and we recorded the data of blood loss, operative time and postoperative complications.

\section{MATERIALS AND METHODS}

Animal preparation

In total, 16 adult male mongrel dogs weighing $12-18 \mathrm{~kg}$ (Centre of Experimental Animal, Medical College of Wuhan University, Wuhan, Hubei, China) were used in this study. All animal experiments were conducted in accordance with the UK Animals (Scientific Procedures) Act of 1986 and its associated guidelines, the EEC Directive of 1986 (86/609/EEC). The study was approved by the Ethical Committee of the Medicine College of Wuhan University. Before the surgical procedure, these dogs were acclimatized and maintained on a standard diet for 1 week.

\section{Circumcision device}

The Harmonic Generator 300 System (Figure 1a), which was purchased from Ethicon Endo-Surgery, Inc. (Cincinnati, OH, USA), and the ACE23E harmonic scalpel (Ethicon Endo-Surgery, Inc.) (Figure 1b) were used in this study. The generator of this system delivers two power levels: minimum (MIN) and maximum (MAX). We adjusted the level of the minimum power to level 3, and the maximum power level was always level 5. In this experiment, we used the minimum power to cut and coagulate the excessive foreskin and avoid damage to the neighbouring structures. 

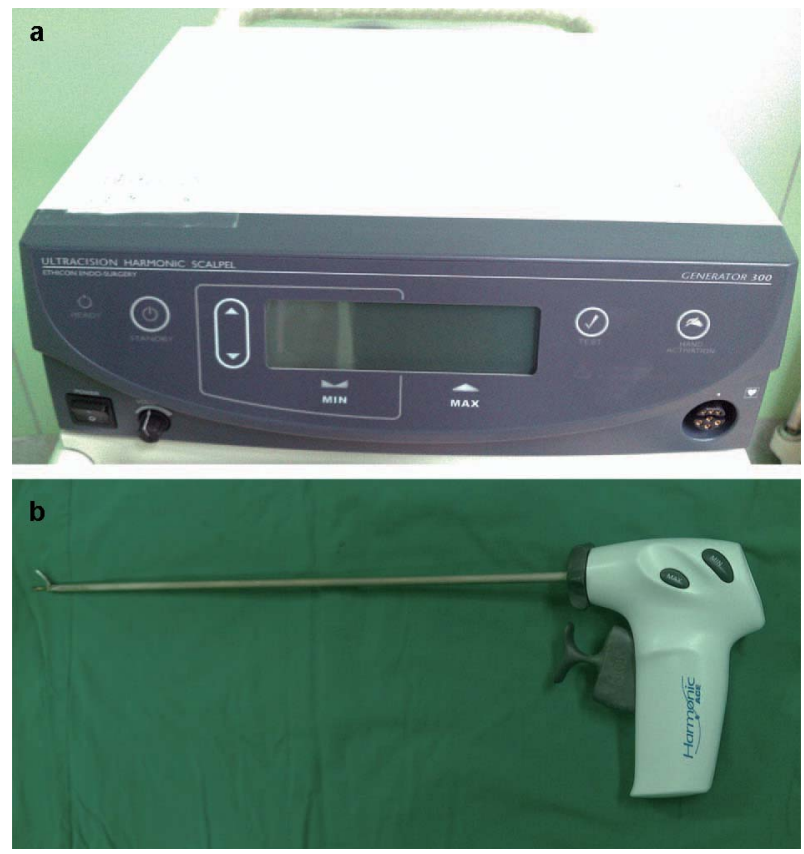

Figure 1 (a) The Harmonic Generator 300 System used in this study. The MIN level was set to level 3 , and the MAX was always set to level 5. (b) The ACE23E harmonic scalpel can be used repeatedly after sterilisation to reduce costs. MAX, maximum; MIN, minimum.

\section{Operative procedure (Supplementary Video)}

After overnight fasting, the animals were anaesthetized with ketamine hydrochloride $\left(10 \mathrm{mg} \mathrm{kg}^{-1}\right.$, i.m.). We used a certain technique as below. We prepared the skin for better visualisation around the penis. After retracting the foreskin and removing all smegma and debris from beneath, we marked the cut level of the preputial skin and fixed it at four points with haemostatic forceps. Then, we made a vertical incision with the UHS in the dorsal aspect of the penis (Figure 2a).
Another vertical incision in the ventral aspect of the penis was made symmetrically (Figure 2b), followed by cutting and welding at the cut level in all cases and addressing all bleeding points with the UHS (Figure 2c and 2d). Because the characteristics of the UHS enabled the ability to weld, we did not use any sutures for the dogs.

In the conventional group, a dissection suturing technique was used. After a circumferential incision along the cut level of the preputial skin, the foreskin was carefully excised to expose the glans. Then, bleeding around the incision was treated by ligation, and gauze was used to compress the wound. The incision was sutured with a $4 / 0$ chromic suture. Finally, we applied povidone iodine over the incision in both the UHS and conventional groups. One week postoperatively, the dogs were anaesthetized with ketamine hydrochloride again, and we examined the recovery and the aesthetic result of the incision in the UHS group.

\section{RESULTS}

\section{Operative data}

Eight dogs underwent circumcision with the UHS, and eight others underwent circumcision with the conventional method (control group). The median operation time was $5.1 \mathrm{~min}$ for the UHS group and $35.5 \mathrm{~min}$ for the conventional group $(P<0.01)$. The median blood loss was less than $2 \mathrm{ml}$ for the UHS group and $15 \mathrm{ml}$ for the conventional group (Table 1).

\section{Postoperative complications}

In the UHS group, there was no moderate oedema, infection or bleeding of the incision or wound dehiscence, and the postoperative complications included only one case of mild oedema. In the conventional group, the postoperative complications included two cases of mild oedema, one infection of the incision and one case of bleeding of the incision (Table 2).

\section{Postoperative effect of the circumcision on the dogs}

The device appears to work satisfactorily, and the postoperative incision is shown in Figure 2e. On the seventh postoperative day,
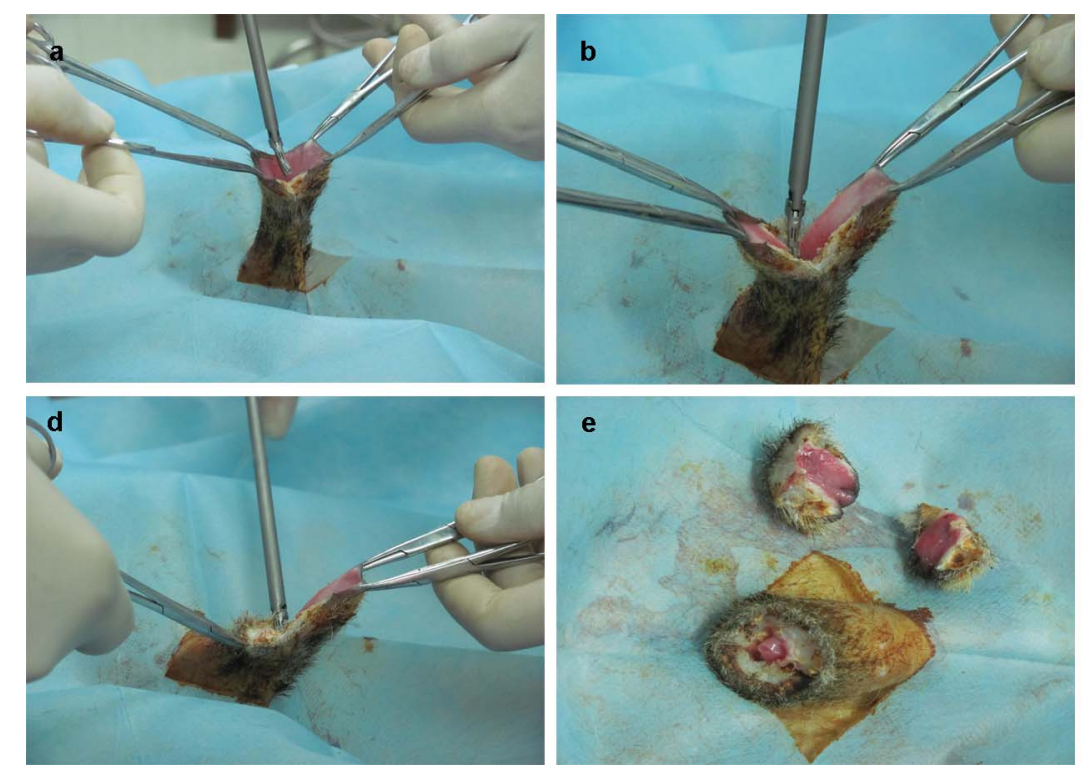
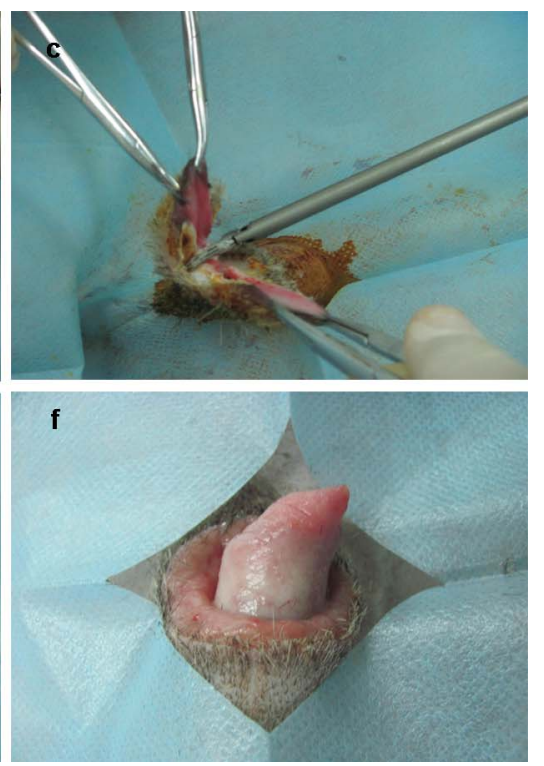

Figure 2 The circumcision procedure using the UHS and the images of the postoperative effects. (a) A vertical incision with the UHS in the dorsal aspect of the penis was made. (b) A vertical incision in the ventral aspect of the penis was made. (c, d) Excessive foreskin was cut, and the incision was welded. (e) Postoperative effects of the circumcision with the UHS. (f) Recovery effects on the seventh postoperative day. UHS, ultrasonic harmonic scalpel. 
Table 1 Postoperative parameters of the UHS group and the conventional group

\begin{tabular}{lcc}
\hline Postoperative parameter & UHS group & Conventional group \\
\hline Mean operation time (min) & 5.1 & 35.5 \\
Mean blood loss $(\mathrm{ml})$ & $<2$ & 15 \\
\hline
\end{tabular}

Abbreviation: UHS, ultrasonic harmonic scalpel.

the site of the incision was smooth, and no scar was apparent (Figure 2f). The glans was fully exposed with no foreskin covering it.

\section{DISCUSSION}

In recent years, numerous techniques for male circumcision have been used, ${ }^{11-13}$ such as conventional circumcision, the shenghuan disposable minimally invasive circumcision anastomosis device, the plastic clamp technique and the Plastibell device. Circumcision is a simple operation, but the techniques of circumcision, regardless of which is selected, have the risk of postoperative complications. The current complication rate for adult male circumcision is approximately $2 \%$ $4 \%$ under optimal conditions ${ }^{9,14,15}$ but is much higher in resourcepoor settings and when circumcision is performed by inexperienced or poorly trained practitioners. Therefore, a safe and effective circumcision technique with fewer associated complications is essential for patients.

The UHS is widely used in laparoscopic and open surgery. The mechanism by which the UHS coagulates and cuts tissues is the conversion of an ultrasonic wave into high-frequency mechanical energy. The cutting surfaces can heat and denature tissue into a coagulum, which prevents blood loss. The temperature in operation is low, at 50 $100{ }^{\circ} \mathrm{C}$, and the risk of damage to the neighbouring structures is also low. The use of the UHS in circumcision is a novel technique that was shown to be convenient and effective in this pilot study. Compared with conventional circumcision, circumcision with the UHS has two advantages. The first is its simplicity and short operative time; the mean operative time was $5.1 \mathrm{~min}$ compared with the $35.5 \mathrm{~min}$ of conventional circumcision. Second, the application of the UHS in circumcision has fewer complications than the conventional scalpel method. In this study, the use of the UHS resulted in reduced blood loss. None of the dogs in the UHS group had an infection or wound dehiscence, and there was only one case of mild oedema. In addition, because of the characteristics of the UHS, circumcision performed with a UHS becomes a sutureless, scalpel-free operation. Circumcision with UHS will also be safe for surgeons in areas with high HIV transmission risks.

Another technique using the Plastibell device is known for its simplicity and convenience. ${ }^{16,17}$ The Plastibell device has been established as an acceptable means of performing circumcision and is the most frequently used circumcision device in the world. ${ }^{18}$ An obvious

Table 2 Numbers of postoperative complications in the UHS group and the conventional group

\begin{tabular}{lcc}
\hline Complications & $\begin{array}{c}\text { No. for UHS group } \\
(\mathrm{n}=8)\end{array}$ & $\begin{array}{c}\text { No. for conventional group } \\
(\mathrm{n}=8)\end{array}$ \\
\hline Mild oedema & 1 & 2 \\
Infection & 0 & 1 \\
Bleeding of the incision & 0 & 1 \\
Wound dehiscence & 0 & 0 \\
\hline
\end{tabular}

Abbreviation: UHS, ultrasonic harmonic scalpel. advantage of using the Plastibell device is the brief surgery time. ${ }^{19}$ The speed and simplicity of the technique could make Plastibell device circumcision a nice option for use in resource-poor areas. However, compared with the UHS, the Plastibell device is more suited to infants and small children. In teenagers and adults, erections can cause the device to move, thereby causing problems, and the device can even be flung off. Another uncomfortable aspect is that the Plastibell device has to be worn for several days. Because an operation is involved, there are also some common complications of the Plastibell device, including the delayed separation of the ring, bleeding, localized superficial infection and proximal migration of the ring. Furthermore, some unusual complications, such as ischaemic glans penis, ${ }^{20}$ urine retention $^{21}$ and grievous penile injury, ${ }^{22}$ may occur. Inappropriate size of the Plastibell device is also a problem, and choosing a suitable size for every patient increases the complexity of its use. Fortunately, the UHS has similar advantages to the Plastibell device while avoiding its deficiencies.

Kaye et al. ${ }^{23}$ reported the combination of electrocautery and 2-octyl cyanoacrylate for circumcision as a safe, efficient, financially beneficial, cosmetically appealing alternative to traditional circumcision performed with scalpel and sutures. However, the UHS has an obvious advantage compared with the electrocautery technique. The operating temperature of the electric knife is $100-200{ }^{\circ} \mathrm{C}$, and cauterisation damage can spread $5 \mathrm{~mm}$ into the surrounding tissue. The working temperature of the harmonic scalpel is less than $100{ }^{\circ} \mathrm{C}$, and the energy spread does not exceed $1 \mathrm{~mm}$. Furthermore, the absence of current through the body makes the surgery using UHS safer.

Our pilot study of circumcision in dogs indicates that the application of the UHS in circumcision may be a novel technique to treat patients with phimosis and excessive foreskin. This technique will also be suitable for men who wish to be circumcised for cosmetic or other personal reasons. A minimum power setting of level 3 was used to cut and coagulate the tissue, and whether the other levels will have better effects remains unknown. In addition, the limitation of the UHS applied to male circumcision is that the technique requires the Harmonic Generator 300 System and a harmonic scalpel, which are expensive if bought solely for the procedure of circumcision. However, if the UHS is also used in laparoscopic surgery and the ultrasonic scalpel is reused after sterilisation, the costs of the circumcision will be reduced. Compared with conventional circumcision, the mean operation time is reduced approximately sixfold in the UHS group, meaning that more patients can undergo the procedure within a specific time frame. With the development of urology, the cost of the UHS will decrease drastically. Taking these findings together, circumcision with the UHS requires further research before being widely used in humans.

In conclusion, circumcision with the UHS is a novel technique to treat patients with phimosis and excessive foreskin, and this method has a shorter operative time, less blood loss and fewer complications than the conventional scalpel method. Our current small animal study provides a basis for embarking on a larger-scale clinical trial of the UHS.

\section{AUTHOR CONTRIBUTIONS}

MP and ZM participated in the design of the study, performed the operation and drafted the manuscript. ZHY participated in the design of the study and performed the statistical analysis. XHW conceived the study, participated in its design and coordination and helped draft the manuscript. All authors read and approved the final manuscript. 
neg

Ultrasonic harmonic scalpel for circumcision using dogs

M Peng et al

96

COMPETING FINANCIAL INTERESTS

The authors have no competing financial interests to declare.

ACKNOWLEDGMENTS

The study was supported by the Science and Technology Research Project of Wuhan (No. 200960223068) and Fundamental Research Funds for the Central Universities (No. 2012303020211).

Supplementary Information accompanies the paper on Asian Journal of Andrology's website (http://www.nature.com/aja).

1 Schoen EJ. The status of circumcision of newborns. N Engl J Med 1990; 322: 1308-12.

2 Wiswell TE, Fencer HL, Welsh CA, Chamberlain JL. Circumcision in children beyond the neonatal period. Pediatrics 1993; 92: 791-3.

3 World Health Organization/UNAIDS. Male circumcision: global trends and determinants of prevalence, safety and acceptability. World Health Organization, Geneva, Switzerland, 2007. http://whqlibdoc.who.int/publications/2007/9789241596169_eng.pdf.

4 Meeting the demand for male circumcision: a report of a workshop. Proceedings of the Meeting of The Forum for Collaborative HIV Research; 13-14 March 2008; Kampala, Uganda.

5 Kochen M, McCurdy S. Circumcision and the risk of cancer of the penis. A life-table analysis. Am J Dis Child 1980; 134: 484-6.

6 Craig JC, Grigor WG, Knight JF. Acute obstructive uropathy-a rare complication of circumcision. Eur J Pediatr 1994; 153: 369-71.

7 Brewer DD, Potterat JJ, Brody S. Male circumcision in HIV prevention. Lancet 2007; 369: 1597.

8 Denniston GC, Hill G. Male circumcision in HIV prevention. Lancet 2007; 369: 1598.

9 Hargreaves S. $60 \%$ reduction in HIV risk with male circumcision, says WHO. Lancet Infect Dis 2007; 7: 313.
10 Hargreave T. Male circumcision: towards a World Health Organisation normative practice in resource limited settings. Asian J Androl 2010; 12: 628-38.

11 Mousavi SA, Salehifar E. Circumcision complications associated with the Plastibell device and conventional dissection surgery: a trial of 586 infants of ages up to 12 months. Adv Ural 2008; 2008: 606123.

12 Peng YF, Chen Y, Wang GY, Wang SQ, Ja C et al. Clinical application of a new device for minimally invasive circumcision. Asian J Androl 2008; 10: 447-54.

13 Seel FM, Demirelli M, Pekan H. Mass circumcision with a novel plastic clamp technique. Urology 2011; 78: 174-9.

14 Krieger JN, Bailey RC, Opeya J, Ayieko B, Opiyo F et al. Adult male circumcision: results of a standardized procedure in Kisumu District, Kenya. BJU Int 2005; 96: 1109-13.

15 Krieger JN, Bailey RC, Opeya JC, Ayieko BO, Opiyo FA et al. Adult male circumcision outcomes: experience in a developing country setting. Ural Int 2007; 78: 235-40.

16 Kazem MM, Mehdi AZ, Golrasteh KZ, Behzad FZ. Comparative evaluation of two techniques of hemostasis in neonatal circumcision using the Plastibell device. J Pediatr Urol 2010; 6: 258-60.

17 Moose FA, Khan FW, Roo MH. Comparison of complications of circumcision by 'Plastibell device technique' in male neonate and infants. J Pax Med Assoc 2010; 60: 664-7.

18 Mousavi SA, Mohammadjafari H. Circumcision with the plastibell device in hooded prepuce or glanular hypospadias. Adv Urol 2009; 2009: 864816.

19 Morris BJ, Ely C. Male circumcision: an appraisal of current instrumentation. In: Fazel-Rezai R, editor. Biomedical Engineering -From Theory to Applications. Rijeka: InTech; 2011. pp315-54.

20 Tzeng YS, Tang SH, Mend E, Lin TF, Sun GH. Ischemic glans penis after circumcision. Asian J Androl 2004; 6: 161-3.

21 Mihssin N, Moorthy K, Houghton PW. Retention of urine: an unusual complication of the Plastibell device. BJU Int 1999; 84: 745.

22 Bode $\mathrm{CO}$, Ikhisemojie S, Ademuyiwa AO. Penile injuries from proximal migration of the Plastibell circumcision ring. J Pediatr Ural 2010; 6: 23-7.

23 Kaye JD, Kalisvaart JF, Luda SP, Elmore JM, Cerwinka WH et al. Sutureless and scalpel-free circumcision -more rapid, less expensive and better? J Ural 2010; 184: 1758-62.

Asian Journal of Andrology 